\title{
ESTUDIO FITOSOCIOLÓGICO Y EVALUACIÓN DEL BANCO DE MALEZAS DEL SUELO EN TRES FINCAS MAICERAS DEL ESTADO PORTUGUESA, VENEZUELA
}

\author{
Sandra Torres ${ }^{1}$ y Aída Ortiz ${ }^{1}$
}

RESUMEN

\begin{abstract}
Los estudios fitosociológicos (EF) y bancos de semillas de malezas del suelo (BSMS) son una herramienta para el manejo de las malezas. El objetivo de esta investigación fue realizar un EF y evaluar el BSMS en tres fincas maiceras del estado Portuguesa, Venezuela, determinándose el índice de valor de importancia (IVI) y plántulas por superficie. Se encontraron diferencias entre la composición florística entre fincas y estudios realizados, detectándose un mayor número de especies en el EF. Las especies con mayor IVI en la finca 1 fueron Ludwigia decurrens Walter, Eclipta prostrata (L.) L., Euphorbia heterophylla L. y Amaranthus dubius Mart. ex Thell., mientras que en el BSMS las de más altas densidades fueron Torenia crustacea (L.) Cham. \& Schltd., L. decurrens y Cyperus esculentus L., con 1809,95; 434,39 y 253,39 plántulas $\cdot \mathrm{m}^{-2}$, respectivamente. En la finca 2, Rottboellia cochinchinensis (Lour.) W.D. Clayton, E. prostrata, Eleusine indica (L.) Gaertn y Helianthus annuus L. tuvieron los más altos IVI, y en el BSMS las especies T. crustaceae, Ammannia latifolia L. y L. decurrens las densidades más altas $(389,14 ; 181,00$ y 126,70 plántulas $\cdot \mathrm{m}^{-2}$, respectivamente). En la finca 3, las malezas con mayores IVI fueron Murdannia nudiflora (L.) Brenan, Cyperus rotundus L. y E. indica, y el BSMS estuvo conformado principalmente por C. rotundus y E. indica con 687,78 y 171,95 plántulas $\cdot \mathrm{m}^{-2}$, respectivamente. En total, en los dos estudios se registraron 32 especies de malezas en 21 familias botánicas. Palabras clave adicionales: Emergencia, manejo de malezas, valor de importancia
\end{abstract}

\begin{abstract}
Phytosociological study and soil weed banks in three corn farms of Portuguese State, Venezuela Phytosociological studies (PS) and soil weed seed banks (SWSB) are important tools for weed management. The objective of this investigation was to perform a PS and to evaluate the SWSB in corn farms. Three farms were sampled and the importance value index (IVI) and number of seedlings per surface area were evaluated. Differences were found in the floristic composition among the farms and the studies carried out. In farm 1 a greater number of species in PE was detected. The species with the highest IVI were Ludwigia decurrens Walter, Eclipta prostrata (L.) L., Euphorbia heterophylla L. and Amaranthus dubius Mart. ex Thell., while in the SWSB those with the highest densities were Torenia crustacea (L.) Cham. \& Schltd., L. decurrens and Cyperus esculentus L., with $1809.95,434.39$ and 253.39 seedlings $\cdot \mathrm{m}^{-2}$, respectively. In farm 2 , Rottboellia cochinchinensis (Lour.) W.D. Clayton, E. prostrata, Eleusine indica (L.) Gaertn and Helianthus annuus L., had the highest IVI, and in the SWSB, the species T. crustaceae, Ammannia latifolia $\mathrm{L}$. and L. decurrens had the highest densities, with $389.14,181.0$ and $126.7 \mathrm{seedling} \cdot \mathrm{m}^{-2}$, respectively. In farm 3, the weeds with the highest IVI were Murdannia nudiflora (L.) Brenan, Cyperus rotundus L. and E. indica, while the SWSB consisted mainly of C. rotundus and E. indica con 687.78 and 171.95 seedlings $\mathrm{m}^{-2}$, respectively. In total, 32 species were recorded in 21 botanical families in the two studies carried out.
\end{abstract}

Additional keywords: Emergence, importance value, weed management

\section{INTRODUCCIÓN}

El maíz es el primer cereal en producción en Venezuela (FAO, 2021), que históricamente ha tenido un papel fundamental en la alimentación de la población, con un consumo de harina de 18,63 kg per cápita en 2017 (Sequín y Sosa, 2019). En el cultivo de maíz en Venezuela, el control de

Recibido: Marzo 29, 2021

${ }^{1}$ Dpto. de Agronomía, Facultad de Agronomía, Universidad Central de Venezuela (UCV). Maracay, Venezuela e-mail: sandra.america.13@gmail.com; aidaortizd@gmail.com (autora de correspondencia) malezas ha sido considerado prioridad para evitar niveles de infestación que afecten su productividad.

A diferencia de otras plagas, las malezas comparten un nivel trófico similar al cultivo, al competir por recursos escasos y causan enormes pérdidas en el rendimiento de los cultivos (Ramesh et al., 2017). Asimismo, las malezas 
generan poblaciones con densidad y distribución heterogénea, lo que dificulta su manejo (Partel et al., 2019).

En los lotes de siembra se producen cambios en la dinámica de malezas asociadas a los cultivos, debido a la interacción entre la genética de las especies (herencia), oferta ambiental, manejo agronómico y vectores que dispersen semillas (Cousens y Mortimer, 1995). El primer pilar fundamental para el manejo integrado de malezas en los cultivos es la identificación de especies y establecimiento de su importancia (Fuentes, 2010). Los estudios fitosociológicos proporcionan una fotografía momentánea de la composición, estructura y distribución de las especies de plantas en una comunidad determinada utilizando datos de frecuencia de aparición, densidad y abundancia a partir de los cuales se estiman índices como el valor de importancia y coeficiente de similitud, que permiten caracterizar a la vegetación (Booth et al., 2003; Erasmo et al., 2004).

A pesar de los esfuerzos para eliminar las malezas, éstas continúan reproduciéndose y su persistencia depende del banco de semillas del suelo (Schwartz-Lazaro y Copes, 2019). El banco de semillas es una reserva del pool genético viable de diásporas no germinadas en un hábitat determinado, constituido por un banco temporal y otro persistente (Delouche et al., 2007).

Las semillas del banco temporal solo germinan durante un ciclo o no sobreviven durante la segunda estación climática, mientras que las del banco persistente duran más de un ciclo o emergen en la segunda época (Thompson y Grime, 1979). En los países templados es fácil separar ambos bancos, por el contrario, en el trópico es difícil representarlos (Baskin y Baskin, 2001).

Las especies de malezas que forman bancos de semillas persistentes son las que ocasionarán más problemas en el manejo de malezas en el futuro. La persistencia de semillas viables en el banco de semillas del suelo depende de una amplia gama de factores que interactúan, como las prácticas de producción y las condiciones ambientales (Korres et al., 2018). Estos factores incluyen poder de germinación, latencia de la semilla, longevidad (Delouche et al., 2007), tamaño de la semilla, edad fisiológica, depredación, descomposición microbiana, condiciones ambientales, profundidad del entierro, duración del entierro y labranza (Liebman et al., 2001). La longevidad de las semillas en el suelo es el factor más determinante para el éxito de las generaciones futuras (Conn et al., 2006).

La comprensión de la dinámica de los bancos de semillas del suelo es fundamental para el desarrollo de sistemas de manejo de malezas más eficientes (Buhler et al., 1997). No obstante, la mayoría de la metodología utilizada en los artículos publicados sobre banco de semillas de malezas del suelo está determinada por los recursos disponibles para el experimento, y lleva mucho tiempo debido a la dificultad de identificar semillas y plántulas a diferencia de hacerlo en plantas adultas con flores (Mahé et al., 2021).

Los estudios sobre bancos de semillas de malezas en el suelo se han utilizado para comparar tratamientos sobre control de maleza (Dey et al., 2018), caracterizar las malezas de agroecosistemas representativos de regiones (Jim et al., 2020), cuantificar la densidad y composición de la flora asociada a cultivos (Forte et al., 2018), contrastar sistemas de labranza y fertilización (Fracchiolla et al., 2018; Santín-Montanyá et al., 2018), y evaluar el potencial de restauración de los bancos de semillas del suelo como un componente importante de la resiliencia de comunidades (Kiss et al., 2018).

El objetivo de esta investigación fue realizar un estudio fitosociológico y evaluar el banco de semilla de malezas del suelo en lotes de tres fincas productoras de maíz del estado Portuguesa para que sea considerado en la formulación de un programa de manejo de plagas por parte de la Asociación Nacional de Cultivadores Agrícolas (ANCA).

\section{MATERIALES Y MÉTODOS}

Se seleccionaron tres fincas $(\mathrm{F} 1, \mathrm{~F} 2$ y $\mathrm{F} 3)$ representativas del programa de siembra de maíz de la Asociación Nacional de Cultivadores Agrícolas (ANCA), ubicadas en los municipios Araure, Turén y Santa Rosalía del estado Portuguesa (Cuadro 1). La temperatura presentó un promedio anual de 27,2 ${ }^{\circ} \mathrm{C}$ y la precipitación total una media de $1538 \mathrm{~mm}$.

Estudio fitosociológico. El número de puntos evaluados se determinó siguiendo un muestreo no probabilístico por conveniencia; en este caso se hicieron cinco muestreos con una cuadrícula de 1 
x $1 \mathrm{~m}$ en cada finca, siguiendo un patrón en zigzag. Se analizaron 15 muestras en total y se cuantificó el número de especies e individuos en cada cuadrícula.

El muestreo se realizó en junio 2016 antes de la siembra del cultivo de maíz.

Cuadro 1. Ubicación, características y área de siembra de las fincas muestreadas en el estado Portuguesa

\begin{tabular}{cccclc}
\hline $\begin{array}{c}\text { Finca } \\
\text { (F) }\end{array}$ & Municipio & Coordenadas & $\begin{array}{c}\text { Superficie de } \\
\text { siembra (ha) }\end{array}$ & \multicolumn{1}{c}{ Suelo } & \multicolumn{2}{c}{$\begin{array}{c}\text { Cultivo de Sistema de } \\
\text { rotación }\end{array}$} \\
\hline F1 labranza
\end{tabular}

Alrededor de cada punto de muestreo se hizo un inventario de la vegetación y descripción para determinar la composición de la comunidad, recolectándose especímenes botánicos para su posterior identificación taxonómica, los cuales se acondicionaron y se enviaron al Herbario Nacional de Venezuela (VEN), de la Universidad Central de Venezuela. En la elaboración del inventario de malezas se utilizó el código de cada especie citado en la plataforma virtual EPPO Global Database (https://gd.eppo.int/).

La determinación taxonómica se efectuó por comparación con la colección del Herbario, revisión de bibliografía especializada (Kissmann y Groth, 1999; Lorenzi, 2000; Bryson y DeFelice, 2009), manejo de claves taxonómicas (Zardini y Raven, 1992; Carrizo y Sobrero, 2001; Carranza, 2007) y consulta de tipos nomenclaturales a través de plataformas virtuales The Plant List (http://www.theplantlist.org/), Tropicos.org (http: //www.tropicos.org/) y US Department of Agriculture (https://plants.sc.egov.usda.gov /java/).

La evaluación de la contribución de las especies halladas en las fincas se hizo calculando los valores absolutos y relativos de las variables densidad, frecuencia, abundancia (cobertura) y valor de importancia, con las siguientes fórmulas (Quintero et al., 2021):

$\mathrm{D}=$ número total de individuos de la especie $/ \mathrm{m}^{2}$

$\mathrm{DR}=$ número total de individuos de la especie/total de individuos en la muestra x 100

$\mathrm{F}=$ número de cuadrículas donde se encontró la especie/número total de cuadrículas

$\mathrm{FR}=$ frecuencia de la especie/frecuencia de todas las especies $\mathrm{x} 100$
A = La cuadrícula de 100 x $100 \mathrm{~cm}$ se dividió con tres cuerdas de nylon en cada lado resultando en 16 sub-cuadros con un área de $0,0625 \mathrm{~m}^{2}$, es decir, cada uno representó el 6,25\% del total.

$\mathrm{AR}=$ Abundancia de la especie/abundancia de todas las especies x 100

Índice valor de importancia $(\mathrm{IVI})=\mathrm{DR}+\mathrm{FR}+\mathrm{AR}$.

Banco de malezas del suelo. Se realizó un muestreo después de la cosecha de maíz en noviembre 2015, usando un patrón diagonal doble en cada finca y se seleccionaron 5 puntos al azar enmarcados dentro de las diagonales de cada una. En cada punto se utilizó un toma-muestra que permitió colectar un área de suelo de $221 \mathrm{~m}^{2}$ a una profundidad de $10 \mathrm{~cm}$, a fin de determinar el banco de malezas (Ortiz et al., 2009).

Se recolectó un total de 15 muestras de suelo, con un peso promedio de $3 \mathrm{~kg}$ cada una. Éstas fueron trasladadas al Laboratorio de Malezas de FAGRO-UCV para su análisis. Adicionalmente, en cada finca se tomaron muestras de suelo para posterior análisis en el Laboratorio de Suelos de la Universidad Nacional Rómulo Gallegos en San Juan de los Morros, estado Guárico.

La variable número de plántulas por superficie se determinó según la metodología de Forcella et al. (2004), para ello las muestras de suelo colectadas se colocaron en bandejas de 17 x 13 x $15 \mathrm{~cm}$ de largo, ancho y profundidad, respectivamente, las cuales se ubicaron en un invernadero donde se regaron continuamente para inducir la emergencia de las malezas. Posteriormente, cada 7 días por 8 veces se cuantificó el número de plántulas de malezas por 
especie que emergieron en estas bandejas. Estas plántulas se identificaron por medio de manuales para malezas gramíneas y ciperáceas (Lorenzi, 2000; Kissmann y Groth, 1997); dicotiledóneas (Kissmann y Groth, 1999) y acuáticas (Kissmann, 1997). Una vez identificadas y contadas las plántulas de malezas emergidas en las bandejas con un área de $0,0221 \mathrm{~m}^{-2}$, éstas se relacionaron a $1 \mathrm{~m}^{2}$. La emergencia se presenta mediante estadística descriptiva con promedios y dispersión.

\section{RESULTADOS Y DISCUSIÓN}

Estudio fitosociológico. La comunidad florística de las tres fincas maiceras según el EF estuvo representada por 17 familias, 26 géneros y 28 especies (20 dicotiledóneas y 13 monocotiledóneas) (Cuadro 2). Las familias con mayor número de especies dentro de la comunidad fueron Asteraceae, Cyperaceae y Poaceae, con cuatro especies cada una. El género que presentó hasta tres especies fue Cyperus. Las malezas en común que se encontraron en las tres fincas evaluadas fueron $A$. dubius, $C$. rotundus, $E$. prostrata y $E$. heterophyla, el resto estuvo presente en una o dos fincas.

Se destaca que en el estudio se hallaron las siguientes cuatro especies, consideradas dentro de las 18 más difíciles de controlar en el mundo: $C$. rotundus, $E$. indica, $S$. halepense y $R$. cochinchinensis (Holm et al., 1977).

El índice de valor de importancia (IVI) de las malezas varió en cada finca, encontrándose que en F1 las especies con IVI mayor al $20 \%$ fueron $L$. decurrens, E. postrata, E. heterophylla, A. dubius y $M$. nudiflora; en F2 $R$. cochinchinensis, $E$. prostrata, E. indica y $H$. annuus y en F3 $M$. nudiflora, $C$. rotundus, E. indica, E. heterophylla, C. iria y C. odoratus (Cuadro 3).

Estas diferencias halladas del IVI entre fincas se sustentan en que el método de evaluación fitosociológica hace una evaluación momentánea de la composición florística (Erasmo et al., 2004), y que la emergencia de malezas depende de muchos factores del suelo (Delouche et al., 2007), lo que indica que la infestación de malezas en cada unidad de producción es única.

En las dos fincas con cero labranza se registró mayor número de especies de malezas (17 en cada una) que en la primera finca (10 especies) en la cual se emplearon pases de rastra; esto es consistente con lo reportado por Chovancova et al. (2020), quienes encontraron mayor infestación de malezas en la condición de cero labranza o siembra directa que en la mínima labranza.

Por otra parte, dado que M. fasciata, $M$. cannifolia y P. hysterophorus solo se encontraron en la primera finca (caracterizada por el $\mathrm{pH}$ muy bajo del suelo), podría indicar que estas malezas alcanzan el éxito ecológico en suelos fuertemente ácidos.

Cada especie de maleza tiene un rango específico de requisitos ambientales necesarios para la germinación de sus semillas (Baskin y Baskin, 2001). Dado que las tres fincas estudiadas tienen diferentes condiciones de acidez del suelo (incluso, reacción alcalina en un caso) (Cuadro 1) y que las especies $A$. dubius, $C$. rotundus y $E$. prostrata se encontraron en todas ellas, es indicio que estas especies de malezas están adaptadas a un amplio rango de $\mathrm{pH}$.

De igual forma, cuando los suelos presentan condiciones de bajo intercambio gaseoso, tales como los suelos arcillosos de la primera finca (Cuadro 1), se produce un ambiente de hipoxia que favorece la generación de metabolitos en la semilla que pueden inhibir su germinación (Benvenuti y Mazzoncini, 2019). Esta condición, sumado al hecho que la referida finca fue la única que incluyó labranza con pases de rastra y el consiguiente enterramiento de las semillas, pudiera explicar la menor incidencia de malezas que ocurrió allí.

En el presente trabajo se registró por primera vez que la especie $M$. cannifolia es maleza en el cultivo de maíz en Venezuela, una planta perenne de reproducción principalmente asexual a través de rizomas o tubérculos (Andersson, 1998), que se encuentra principalmente en ambientes de sabana, pero también en hábitats húmedos y sombreados (Vieira et al., 2012). Se conoce una sola especie en el género Myrosma (Luna et al., 2020).

Aunque las cifras del valor de importancia no son directamente comparables entre distintos estudios (Goff y Cottam, 1967), en Venezuela se han señalado diferentes especies coincidentes o no con este estudio en la comunidad de malezas que afectan al cultivo de maíz; por ejemplo, en siembra directa de maíz en la localidad de Memo, en las fincas agropecuarias Tierra Nueva y Fundo San Rafael, ubicados en el estado Guárico, Venezuela, se determinó que las especies $S$. halepense, $E$. 
heterophylla, P. hysterophorus, $C$ rotundus, $E$. prostrata, $C$. iria y $R$. cochinchinensis, coincidentes con este estudio tuvieron un alto IVI (C. Zambrano, UCV. Comunicación personal). En otro estudio realizado en el cultivo de maíz con riego en el municipio Leonardo Infante, estado Guárico, Venezuela, las especies Digitaria sanguinalis (L.) Scop. (no reportada en esta investigación) y $C$. rotundus (corocillo) predominaron de acuerdo con el análisis fitosociológico (Gámez et al., 2011).

De las especies Poaceae detectadas en la comunidad asociada al cultivo de maíz en Portuguesa, E. indica actualmente presenta problemas de control con glifosato. E. indica ha evolucionado en resistencia a glifosato en otros países (Takano et al., 2019; Chen et al., 2020). No obstante, se ha logrado un excelente control de $E$. indica con topramezone + atrazina y baja eficacia con nicosulfuron (Uztarroz, 2016).

Cuadro 2. Inventario de clases, familia, géneros y especies encontradas en los muestreos de estudios fitosociológicos y bancos de semillas de malezas en el suelo de tres fincas maiceras evaluadas en el estado Portuguesa, Venezuela

\begin{tabular}{|c|c|c|c|c|c|}
\hline Dicotiledóneas/Familia/Especie & $\begin{array}{l}\text { Código } \\
\text { EPPO }\end{array}$ & $\begin{array}{l}\text { Tipo de } \\
\text { planta }\end{array}$ & Dicotiledóneas/Familia/Género & $\begin{array}{l}\text { Código } \\
\text { EPPO }\end{array}$ & $\begin{array}{l}\text { Tipo de } \\
\text { planta }\end{array}$ \\
\hline Amaranthacea & & & Nyctaginaceae & & \\
\hline Amaranthus dubius Mart. ex Thell. & AMADU & Anual & Boerhavia erecta L. & BOEER & $\begin{array}{l}\text { Anual/ } \\
\text { Perenne }\end{array}$ \\
\hline Asteraceae & & & Onagraceae & & \\
\hline Eclipta prostrata (L.) L. & ECLAL & Anual & Ludwigia decurrens Walter & IUSDE & Anual \\
\hline Helianthus annuus L. & HELAN & Anual & Scrophulariaceae & & \\
\hline Parthenium hysterophorus L. & PTNHY & Anual & $\begin{array}{l}\text { Mecardonia procumbens (Mill.) } \\
\text { Small }\end{array}$ & PAGDI & Anual \\
\hline Tridax procumbens $\mathrm{L}$. & TRQPR & $\begin{array}{l}\text { Anual/ } \\
\text { Perenne }\end{array}$ & Zigophylaceae & & \\
\hline Boraginaceae & & & $\begin{array}{l}\text { Kallstroemia maxima }(\text { L.) Hook y } \\
\text { Arn }\end{array}$ & KALMA & Anual \\
\hline $\begin{array}{l}\text { Euploca procumbens (Mill.) Diane y } \\
\text { Hilger }\end{array}$ & HEOPR & & & & \\
\hline Cleomaceae & & & Monocotiledóneas/ Familia/Especie & $\begin{array}{l}\text { Código } \\
\text { EPPO }\end{array}$ & $\begin{array}{c}\text { Tipo de } \\
\text { planta }\end{array}$ \\
\hline $\begin{array}{l}\text { Corynandra viscosa (L.) Cochrane y Iltis } \\
\text { Convolvulaceae }\end{array}$ & CLEVI & Anual & $\begin{array}{l}\text { Alismataceae } \\
\text { Limnocharis flava (L.) Buchenau }\end{array}$ & LMNFL & Perenne \\
\hline Ipomoea trifida (Kunth) G. Don. & IPOTC & Anual & Commelinaceae & & \\
\hline Cucurbitaceae & & & Murdannia nudiflora (L.) Brenan & MUDNU & $\begin{array}{l}\text { Anual/ } \\
\text { perenne }\end{array}$ \\
\hline Portulaca oleracea L. & POROL & Anual & Cyperaceae & & \\
\hline Cucurbitaceae & & & Cyperus esculentus L. & CYPES & Perenne \\
\hline Cucumis melo L. & CUMME & Anual & Cyperus iria $\mathrm{L}$. & CYPIR & Anual \\
\hline Euphorbiaceae & & & Cyperus odoratus $\mathrm{L}$. & CYPFE & $\begin{array}{l}\text { Anual/ } \\
\text { Perenne }\end{array}$ \\
\hline Chamaesyce hirta (L.) Millsp. & EPHHI & Anual & Cyperus rotundus $\mathrm{L}$. & CYPRO & Perenne \\
\hline $\begin{array}{l}\text { Euphorbia heterophylla } \mathrm{L} . \\
\text { Lamiaceae }\end{array}$ & EPHHL & Anual & $\begin{array}{l}\text { Fimbristylis litorallis Gaudich. } \\
\text { Marantaceae }\end{array}$ & FIMLI & Anual \\
\hline $\begin{array}{l}\text { Mesosphaerum suaveolens (L.) Kuntze } \\
\text { Linderniaceae }\end{array}$ & HPYSU & Perenne & $\begin{array}{l}\text { Myrosma cannifolia } \text { L. f. } \\
\text { Poaceae }\end{array}$ & & Perenne \\
\hline Torenia crustacea (L.) Cham. y Schltd. & LIDCR & $\begin{array}{l}\text { Anual/ } \\
\text { perenne }\end{array}$ & $\begin{array}{l}\text { Dinebra scabra (Nees) P.M. } \\
\text { Peterson y N.Snow }\end{array}$ & LEFSC & Anual \\
\hline Lythraceae & & & Echinochloa colona (L.) Link & ECHCO & Anual \\
\hline Ammannia latifolia $\mathrm{L}$. & AMMLA & Anual & Eleusine indica (L.) Gaertn & ELEIN & Anual \\
\hline Malvaceae & & & $\begin{array}{l}\text { Rottboellia cochinchinensis (Lour.) } \\
\text { W.D. Clayton }\end{array}$ & ROOEX & Anual \\
\hline Malachra fasciata Jacq. & MAAFA & Perenne & Sorghum halepense (L.) Pers. & SORHA & Perenne \\
\hline
\end{tabular}


Adicionalmente se han encontrado problemas en el control malezas en $S$. halepense y $R$. cochinchinensis procedentes del estado Portuguesa, Venezuela, debido a que han evolucionado en resistencia a nicosulfuron, herbicida sistémico postemergente mayormente utilizado en el cultivo de maíz para el control de malezas gramíneas (Delgado et al., 2006; Ortiz et al., 2014).

Cuadro 3. Valores del índice de valor de importancia y sus componentes en tres fincas maiceras evaluadas en el estado Portuguesa, Venezuela

\begin{tabular}{|c|c|c|c|c|c|}
\hline $\begin{array}{l}\text { Fincas (f)/ } \\
\text { Municipios }\end{array}$ & Malezas & $\begin{array}{c}\text { Densidad } \\
\text { relativa }(\%)\end{array}$ & $\begin{array}{l}\text { Frecuencia } \\
\text { relativa }(\%)\end{array}$ & $\begin{array}{l}\text { Abundancia } \\
\text { relativa }(\%)\end{array}$ & $\begin{array}{l}\text { Índice de valor de } \\
\text { importancia }\end{array}$ \\
\hline \multirow{10}{*}{ f1/ Araure } & Ludwigia decurrens Walter & 56,46 & 19,05 & 18,42 & 93,93 \\
\hline & Eclipta prostrata (L.) L. & 21,70 & 14,29 & 21,05 & 57,04 \\
\hline & Euphorbia heterophylla L. & 5,28 & 14,29 & 14,04 & 33,60 \\
\hline & Amaranthus dubius Mart. ex Thell. & 4,19 & 14,29 & 12,72 & 31,20 \\
\hline & Murdannia nudiflora (L.) Brenan & 3,49 & 9,52 & 10,96 & 23,98 \\
\hline & Cyperus rotundus $\mathrm{L}$. & 2,10 & 9,52 & 5,70 & 17,32 \\
\hline & Myrosma cannifolia L. f. & 2,66 & 4,76 & 5,70 & 13,12 \\
\hline & Parthenium hysterophorus L. & 3,28 & 4,76 & 4,39 & 12,43 \\
\hline & Malachra fasciata Jacq. & 0,03 & 4,76 & 4,39 & 9,18 \\
\hline & Sorghum halepense (L.) Pers. & 0,80 & 4,76 & 2,63 & 8,20 \\
\hline \multirow{17}{*}{ f2/ Turén } & Rottboellia cochinchinensis (Lour.) W.D. Clayton & 37,16 & 13,33 & 19,62 & 70,11 \\
\hline & Eclipta prostrata (L.) L. & 25,43 & 10,00 & 15,85 & 51,28 \\
\hline & Eleusine indica (L.) Gaertn & 7,86 & 6,67 & 10,19 & 24,72 \\
\hline & Helianthus annuus L. & 0,46 & 10,00 & 10,94 & 21,40 \\
\hline & Corynandra viscosa $(\mathrm{L}$.$) Cochrane y Iltis$ & 3,34 & 10,00 & 6,42 & 19,76 \\
\hline & Amaranthus dubius Mart. ex Thell. & 0,92 & 10,00 & 8,30 & 19,22 \\
\hline & Chamaesyce hirta (L.) Millsp. & 7,73 & 3,33 & 5,28 & 16,35 \\
\hline & Euphorbia heterophylla L. & 0,39 & 6,67 & 5,28 & 12,34 \\
\hline & Cyperus rotundus $\mathrm{L}$. & 4,26 & 3,33 & 4,53 & 12,12 \\
\hline & Torenia crustacea (L.) Cham. y Schltd. & 5,44 & 3,33 & 3,02 & 11,79 \\
\hline & Ipomoea trifida (Kunth) G. Don. & 1,05 & 3,33 & 6,04 & 10,42 \\
\hline & Mecardonia procumbens (Mill.) Small & 2,23 & 3,33 & 1,13 & 6,69 \\
\hline & Euploca procumbens (Mill.) Diane \& Hilger & 1,77 & 3,33 & 0,75 & 5,86 \\
\hline & Tridax procumbens $\mathrm{L}$. & 1,25 & 3,33 & 0,75 & 5,33 \\
\hline & Kallstroemia maxima (L.) Hook y Arn & 0,26 & 3,33 & 0,75 & 4,35 \\
\hline & Dinebra scabra (Nees) P.M.Peterson y N.Snow & 0,20 & 3,33 & 0,75 & 4,28 \\
\hline & Boerhavia erecta $\mathrm{L}$. & 0,26 & 3,33 & 0,38 & 3,97 \\
\hline \multirow{17}{*}{$\begin{array}{l}\text { f3/Santa } \\
\text { Rosalía }\end{array}$} & Murdannia nudiflora (L.) Brenan & 42,95 & 8,57 & 12,96 & 64,48 \\
\hline & Cyperus rotundus $\mathrm{L}$. & 30,59 & 8,57 & 10,49 & 49,65 \\
\hline & Eleusine indica (L) Gaertn & 12,26 & 11,43 & 8,33 & 32,02 \\
\hline & Euphorbia heterophylla L. & 4,50 & 11,43 & 12,35 & 28,28 \\
\hline & Cyperus iria $\mathrm{L}$. & 0,98 & 8,57 & 12,35 & 21,89 \\
\hline & Cyperus odoratus $\mathrm{L}$. & 2,55 & 11,43 & 7,10 & 21,08 \\
\hline & Amaranthus dubius Mart. ex Thell. & 0,63 & 5,71 & 6,48 & 12,82 \\
\hline & Corynandra viscosa $($ L.) Cochrane y Iltis & 0,38 & 5,71 & 5,86 & 11,96 \\
\hline & Tridax procumbens. $\mathrm{L}$ & 0,59 & 5,71 & 5,56 & 11,86 \\
\hline & Rottboellia cochinchinensis (Lour.) W.D. Clayton & 2,34 & 2,86 & 4,94 & 10,13 \\
\hline & Eclipta prostrata (L.) L. & 0,63 & 2,86 & 4,94 & 8,42 \\
\hline & Fimbristylis litorallis Gaudich. & 0,73 & 2,86 & 3,40 & 6,99 \\
\hline & Cucumis melo L. & 0,28 & 2,86 & 2,16 & 5,30 \\
\hline & Ipomoea trifida (Kunth) G. Don. & 0,17 & 2,86 & 1,23 & 4,27 \\
\hline & Chamaesyce hirta (L.) Millsp. & 0,10 & 2,86 & 0,93 & 3,89 \\
\hline & Mesosphaerum suaveolens (L.) Kuntze & 0,28 & 2,86 & 0,62 & 3,75 \\
\hline & Ludwigia decurrens Walter & 0,03 & 2,86 & 0,31 & 3,20 \\
\hline
\end{tabular}


En el manejo de las malezas pertenecientes a la familia Cyperaceae detectadas en el EF, la especie C. rotundus (perenne) resultó ser la más difícil de controlar. Su manejo se enfoca en la reducción de tubérculos y su viabilidad (Webster et al., 2008). C. rotundus es difícil de controlar químicamente debido a la longevidad de los tubérculos (Keeley y Thullen, 1971) y a la falta de eficacia de los herbicidas. Por su parte, $M$. nudiflora, especie de la familia Commelinaceae es una planta perenne, rastrera y estolonífera con muchos tallos, altamente invasora que crece en suelos húmedos y difícil de controlar (Tascón y Fischer, 1997).

Banco de malezas del suelo. En el estudio fitosociológico y banco de semillas de malezas en el suelo (BSMS) se detectaron ocho especies en común, las cuales fueron D. scabra, $A$. dubius, E. indica, L. decurrens, M. nudiflora, $R$. cochinchinensis, $S$. halepense y $T$. crustacea. Por su parte, en cada método de evaluación se hallaron especies no comunes como las 20 especies identificada en el EF y cinco en BSMS, $A$. latifolia, C. esculentus, E. colona, L. flava y $P$. oleraceae (Cuadro 4).

Cuadro 4. Presencia o ausencia de especies detectadas en estudios fitosociológicos y banco de semillas de malezas del suelo en tres fincas maiceras evaluadas en el estado Portuguesa, Venezuela

\begin{tabular}{|c|c|c|c|c|c|c|}
\hline \multirow[b]{2}{*}{ Especie } & \multicolumn{3}{|c|}{ Fitosociológico } & \multicolumn{3}{|c|}{ Banco de semillas } \\
\hline & $\mathrm{F} 1$ & $\mathrm{~F} 2$ & F3 & $\mathrm{F} 1$ & $\mathrm{~F} 2$ & $\mathrm{~F} 3$ \\
\hline Dinebra scabra (Nees) P.M.Peterson y N.Snow & & $\mathrm{X}$ & & & $\mathrm{X}$ & \\
\hline Amaranthus dubius Mart. ex Thell. & $\mathrm{X}$ & $\mathrm{X}$ & $\mathrm{X}$ & $\mathrm{X}$ & $\mathrm{X}$ & $\mathrm{X}$ \\
\hline Eleusine indica (L.) Gaertn & & $\mathrm{X}$ & $\mathrm{X}$ & $\mathrm{X}$ & $\mathrm{X}$ & $\mathrm{X}$ \\
\hline Ludwigia decurrens Walter & $\mathrm{X}$ & & $\mathrm{X}$ & $\mathrm{X}$ & $\mathrm{X}$ & $\mathrm{X}$ \\
\hline Murdannia nudiflora (L.) Brenan & $\mathrm{X}$ & & $\mathrm{X}$ & $\mathrm{X}$ & & \\
\hline Rottboellia cochinchinensis (Lour.) W.D. Clayton & & $\mathrm{X}$ & $\mathrm{X}$ & & $\mathrm{X}$ & $\mathrm{X}$ \\
\hline Sorghum halepense (L.) Pers. & $\mathrm{X}$ & & & & $\mathrm{X}$ & \\
\hline Torenia crustacea (L.) Cham. y Schltd. & & $\mathrm{X}$ & & $\mathrm{X}$ & $\mathrm{X}$ & $\mathrm{X}$ \\
\hline Boerhavia erecta $\mathrm{L}$. & & $\mathrm{X}$ & & & & \\
\hline Chamaesyce hirta (L.) Millsp. & & $\mathrm{X}$ & $\mathrm{X}$ & & & \\
\hline Corynandra viscosa (L.) Cochrane y Iltis & & $\mathrm{X}$ & $\mathrm{X}$ & & & \\
\hline Cucumis melo $\mathrm{L}$. & & & $\mathrm{X}$ & & & \\
\hline Cyperus iria $\mathrm{L}$. & & & $\mathrm{X}$ & & & \\
\hline Cyperus odoratus L. & & & $\mathrm{X}$ & & & \\
\hline Cyperus rotundus $\mathrm{L}$. & $\mathrm{X}$ & $\mathrm{X}$ & $\mathrm{X}$ & & & \\
\hline Eclipta prostrata (L.) L. & $\mathrm{X}$ & $\mathrm{X}$ & $\mathrm{X}$ & & & \\
\hline Euphorbia heterophylla $\mathrm{L}$. & $\mathrm{X}$ & $\mathrm{X}$ & $\mathrm{X}$ & & & \\
\hline Euploca procumbens (Mill.) Diane y Hilger & & $\mathrm{X}$ & & & & \\
\hline Fimbristylis litorallis Gaudich. & & & $\mathrm{X}$ & & & \\
\hline Helianthus аппииs L. & & $\mathrm{X}$ & & & & \\
\hline Ipomoea trifida (Kunth) G. Don. & & $\mathrm{X}$ & $\mathrm{X}$ & & & \\
\hline Kallstroemia maxima (L.) Hook y Arn & & $\mathrm{X}$ & & & & \\
\hline Malachra fasciata Jacq. & $\mathrm{X}$ & & & & & \\
\hline Mecardonia procumbens (Mill.) Small & & $\mathrm{X}$ & & & & \\
\hline Mesosphaerum suaveolens (L.) Kuntze & & & $\mathrm{X}$ & & & \\
\hline Myrosma cannifolia L. f. & $\mathrm{X}$ & & & & & \\
\hline Parthenium hysterophorus $\mathrm{L}$. & $\mathrm{X}$ & & & & & \\
\hline Tridax procumbens. L. & & $\mathrm{X}$ & $\mathrm{X}$ & & & \\
\hline Ammannia latifolia $\mathrm{L}$. & & & & & $\mathrm{X}$ & $\mathrm{X}$ \\
\hline Cyperus esculentus L. & & & & $\mathrm{X}$ & & $\mathrm{X}$ \\
\hline Echinochloa colona (L.) Link & & & & $\mathrm{X}$ & $\mathrm{X}$ & $\mathrm{X}$ \\
\hline Limnocharis flava (L.) Buchenau & & & & & & $\mathrm{X}$ \\
\hline Portulaca oleracea L. & & & & $\mathrm{X}$ & & $\mathrm{X}$ \\
\hline
\end{tabular}

En el Cuadro 5 se registran las especies encontradas en los BSMS en tres fincas del estado
Portuguesa, en Venezuela, para un total de 13 malezas identificadas, repartidas en 9 familias y 
13 géneros.

La familia Poaceae se atribuyó el mayor número de especies con cinco malezas, cuatro de ellas consideradas como las más dañinas del mundo por ser muy difíciles de controlar: $E$. indica, E. colona, $S$. halepense y $R$. cochinchinensis (Holm et al., 1977), el resto de las familias Cyperaceae, Commelinaceae,
Onagraceae, Amaranthaceae, Lytraceae, Cucurbitaceae, Alismataceae y Linderniaceae tuvieron una sola especie. Entre las Monocotiledóneas se encontraron ocho especies, y en las Dicotiledóneas cinco. Las especies comunes en el banco de semillas de malezas del suelo en las tres fincas analizadas fueron E. colona, E. indica, A. dubius, L. decurrens y T. crustacea.

Cuadro 5. Malezas por superficie de suelo encontradas en el banco de semillas a la profundidad de 0-10 $\mathrm{cm}$ en tres fincas maiceras evaluadas en el estado Portuguesa, Venezuela

\begin{tabular}{|c|c|c|c|}
\hline Finca $(\mathrm{F})$ & Especie & Plántulas $\cdot \mathrm{m}^{-2}$ & SD \\
\hline \multirow{8}{*}{$\mathrm{F} 1$} & Torenia crustacea (L.) Cham. y Schltd. & 1809,95 & 853,75 \\
\hline & Ludwigia decurrens Walter & 434,39 & 720,15 \\
\hline & Cyperus esculentus $\mathrm{L}$. & 253,39 & 93,83 \\
\hline & Amaranthus dubius Mart. ex Thell. & 108,60 & 218,42 \\
\hline & Murdannia nudiflora (L.) Brenan & 36,20 & 80,94 \\
\hline & Eleusine indica (L.) Gaertn & 18,10 & 24,78 \\
\hline & Portulaca oleracea $L$. & 18,10 & 24,78 \\
\hline & Echinochloa colona (L.) Link & 9,05 & 20,24 \\
\hline \multirow{9}{*}{$\mathrm{F} 2$} & Torenia crustacea (L.) Cham. y Schltd. & 389,14 & 489,02 \\
\hline & Ammannia latifolia $\mathrm{L}$. & 181,00 & 404,72 \\
\hline & Ludwigia decurrens Walter & 126,70 & 283,30 \\
\hline & Amaranthus dubius Mart. ex Thell. & 63,35 & 75,72 \\
\hline & Rottboellia cochinchinensis (Lour.) W.D. Clayton & 63,35 & 75,72 \\
\hline & Eleusine indica (L.) Gaertn & 54,30 & 59,00 \\
\hline & Sorghum halepense (L.) Pers. & 36,20 & 49,57 \\
\hline & Echinochloa colona (L.) Link & 9,05 & 20,24 \\
\hline & Dinebra scabra (Nees) P.M.Peterson y N.Snow & 9,05 & 20,24 \\
\hline \multirow{10}{*}{ F3 } & Cyperus esculentus $\mathrm{L}$. & 687,78 & 488,81 \\
\hline & Eleusine indica (L.) Gaertn & 171,95 & 59,00 \\
\hline & Ludwigia decurrens Walter & 90,50 & 71,54 \\
\hline & Torenia crustacea (L.) Cham. y Schltd. & 81,45 & 80,94 \\
\hline & Amaranthus dubius Mart. ex Thell. & 27,15 & 40,47 \\
\hline & Ammannia latifolia $\mathrm{L}$. & 27,15 & 24,78 \\
\hline & Portulaca oleracea L. & 27,15 & 40,47 \\
\hline & Echinochloa colona (L.) Link & 18,10 & 40,47 \\
\hline & Limnocharis flava (L.) Buchenau & 18,10 & 40,47 \\
\hline & Rottboellia cochinchinensis (Lour.) W.D. Clayton & 9,05 & 20,24 \\
\hline
\end{tabular}

SD: desviación estándar

En F1 se identificaron ocho especies, hallándose que la de mayor densidad fue $T$. crustacea con 1800,95 plántulas $\cdot \mathrm{m}^{-2}$ (aproximadamente 40 plántulas por bandeja), seguida por $L$. decurrens, $C$. esculentus y $A$. dubius con 434,39; 253,39 y 108,60 plántulas $\cdot \mathrm{m}^{-2}$, respectivamente. El resto de las malezas tuvieron entre 36,20 a 9,05 plántulas $\cdot m^{-2}$. En $F 2$ se detectaron nueve especies, dominando tres dicotiledóneas, $T$. crustaceae, A. latifolia y $L$. 
decurrens con densidades de 389,14; 181,00 y 126,70 plántulas $\cdot \mathrm{m}^{-2}$; no obstante, se hallaron cinco Poaceae y una Amaranthaceae con valores entre 63,5 y 9,05 plántulas $\mathrm{m}^{-2}$. En $\mathrm{F} 3$ se detectaron diez especies, la mayor densidad se halló en $C$. esculentus con 687,78 plántulas $\cdot \mathrm{m}^{-2}$, seguida por $E$. indica con 171,95 plántulas $\cdot \mathrm{m}^{-2}$, mientras que ocho especies entre monocotiledóneas y dicotiledóneas compartieron un rango entre 71,54 y 20,24 plántulas $\cdot \mathrm{m}^{-2}$. Los altos valores de desviación estándar indican que en algunas muestras no se encontraron plántulas, mientras que en otras tuvieron un alto número (Cuadro 5).

La detección de especies en el BSMS fue menor que en el EF, quizás atribuido a que el modelo espacial de las semillas de malezas en el banco del suelo en campos agrícolas, por lo general, sigue un patrón de dispersión agregada (tiende a concentrarse en las cercanías de las plantas madre) (Forcella et al., 2004). Además, la unidad de muestreo del banco de semillas fue más más pequeña que la usada en el estudio fitosociológico, y dado que se hizo el muestreo del BSMS después de la cosecha del maíz, quizás las semillas de algunas especies tuvieron latencia al momento de la evaluación mientras que el EF se hizo antes de sembrar el maíz (momentos diferentes).

En el levantamiento florístico de las tres fincas evaluadas se encontraron 33 especies en 16 familias en la clase dicotiledóneas y 5 en la monocotiledónea. Las familias con mayor número de especies fueron Poaceae, Cyperaceae y Asteraceae (cinco las dos primeras y cuatro la última), seguidas por Euphorbiaceae (dos especies). Los géneros con mayor número de especies fueron Poaceae y Cyperaceae con cinco cada uno. En cuanto con el tipo de plantas se hallaron 20 especies anuales, 7 perennes y 6 anuales o perennes (Cuadro 2).

Aunque la mayoría de las malezas importantes en los cultivos anuales son por lo general plantas anuales, pero las especies más dañinas son perennes debido a que son más difíciles de controlar.

Las plantas perennes desarrollan órganos vegetativos capaces de sobrevivir a períodos desfavorables para el crecimiento. Estos órganos tienen tejidos a partir de los cuales se puedan iniciar nuevas raíces y brotes (nuevas plantas) y otros que almacenan reservas para el metabolismo celular.

La multiplicación vegetativa ocurre espontáneamente en muchas especies perennes como resultado de la división de las estructuras vegetativas por la muerte de los tejidos conectivos, por lo que se desarrollan clones con un número creciente de individuos separados (Håkansson, 1982). En el trópico y subtrópico se encuentran especies rizomatosas perennes como el C. rotundus, C. esculentus y $S$. halepense, halladas en este estudio y que son consideradas dentro de las 18 especies más nocivas del mundo (Holm et al., 1977).

El manejo integrado de malezas es un desafío constante; por ejemplo, se sabe que es importante evitar que se establezcan plántulas vecinas de otras especies como se pudo determinar en un experimento Lolium perenne L. y maíz, debido a que la competencia indujo una acumulación de $\mathrm{H}_{2} \mathrm{O}_{2}$ (indicador de estrés) en la primera hoja y en los tejidos del cuello de la raíz de las plantas del cultivo; lo anterior, atribuido a una baja relación rojo y rojo lejano (R: RL) en el espectro de la radiación solar, lo cual produjo cambios moleculares y fisiológicos que quizás sean la clave del mecanismo real de la pérdida de rendimiento del cultivo, debido a la competencia inter-específica (Afifi y Swanton, 2012). Por ello, se hace necesario el monitoreo y vigilancia de la emergencia en campo con metodologías pertinentes que permitan registrar la proporción de malezas en los bancos de malezas del suelo, una fotografía en cada ciclo de cultivo, de manera de llevar el historial de los lotes de siembra.

En el futuro de la ciencia de las malezas, cuando la oferta de herbicidas sea escasa y aumente la presión social por la conservación del ambiente, se harán más importante los estudios sobre biología básica (genética, evolución y ecología), así como el conocimiento de la tecnología de robótica, inteligencia artificial, sistemas de información geográfica, teledetección y big data para el manejo de las malezas (Westwood et al., 2018).

\section{CONCLUSIONES}

El estudio fitosociológico (EF) y la evaluación del banco de semillas de malezas del suelo (BSMS) en tres fincas maiceras con condiciones 
variables de suelo mostraron diferencias en las especies detectadas, valoraciones en IVI y número de plántulas unidad de superficie, evidenciándose que en una de las fincas las malezas con mayores incidencias fueron las de hojas anchas ( $L$. decurrens, E. postrata y $T$. crustaceae), en la otra la gramínea $R$. cochinchinensis, y en la tercera la Commelinácea $M$. nudiflora y la Ciperácea $C$. esculentus.

En el EF hubo más riqueza de especies que en el BSMS. En ambos estudios se identificaron 33 especies en 21 familias botánicas, con mayor predominancia de la clase dicotiledóneas, las familias Poaceae, Cyperaceae y Asteraceae; el género Cyperus y las plantas anuales. Las especies comunes que se identificaron fueron $T$. crustacea, A. dubius, L. decurrens, E. indica, D. scabra, $R$. cochinchinensis, $S$. halepense y $M$. nudiflora.

\section{AGRADECIMIENTO}

A la Asociación Nacional de Cultivadores Agrícolas (ANCA), especialmente a su gerente Lic. Marisela González por el financiamiento otorgado al proyecto titulado: Evaluación de Malezas del Programa de Producción de Maíz de ANCA. A los productores de maíz Luis Rivero González, Paolo Denuncio Ambrosini y Neris Lobatón por permitir realizar está investigación en sus fincas. A los ingenieros Miguel Brito, Óscar Azuaje y Betzabet Alezone al ayudar en la recolección de muestras. A la profesora Yazmilet Tiberio por su ayuda en el procesamiento de muestras para el estudio fitosociológico. A los especialistas en taxonomía botánica Dr. Shingo Nosawa (Herbario UCV, Caracas-Venezuela) y Dr. Gerardo Aymard (Herbario Universitario PORT, UNELLEZ-Guanare-Venezuela) por su ayuda en la identificación de las malezas.

\section{LITERATURA CITADA}

1. Afifi, M. y C. Swanton. 2012. Early physiological mechanisms of weed competition. Weed Science 60: 542-551.

2. Andersson, L. 1998. Marantaceae. In: K. Kubitzki (ed.). Flowering Plants Monocotyledons. Springer, Berlin, Heidelberg. pp. 278-293.

3. Baskin, C. y J. Baskin. 2001. Germination ecology of seed in the persistent seed bank. In: C. Baskin y J. Baskin (eds.) Seeds Ecology, Biogeography, and Evolution of Dormancy and Germination. Academic Press, New York. pp.133-179.

4. Benvenuti, S. y M. Mazzoncini. 2019. Soil physics involvement in the germination ecology of buried weed seeds. Plants 8(1): 7.

5. Booth, B., S. Murphy y C. Swanton. 2003. Weed ecology in natural and agricultural systems. CABI Publishing, Wallingford.

6. Bryson, C. y M. DeFelice. 2009. Weeds of the South. University of Georgia Press. US.

7. Buhler, D., R. Hartzler, F. Forcella. 1997. Implications of weed seedbank dynamics to weed management. Weed Science 45: 329-336.

8. Carranza, E. 2007. Familia Convolvulaceae. Flora del Bajío y de Regiones Adyacentes. Fascículo 151. Pátzcuaro, Michoacán.

9. Carrizo, E. y Y. Sobrero. 2001. Description of the species of the genus Ipomoea in the irrigated area of Río Dulce, Santiago del Estero, Argentina. Planta Daninha 19(2): 155-161.

10. Chovancova, S., F. Illek y J. Winkler. 2020. The effect of three tillage treatments on weed infestation in maize monoculture. Pak. J. Bot 52(2): 697-701.

11.Chen, J., H. Huang, S. Wei, S, H. Cui, X. Li y C. Zhang. 2020. Glyphosate resistance in Eleusine indica: EPSPS overexpression and P106A mutation evolved in the same individuals. Pesticide Biochemistry and Physiology 164: 203-208.

12.Conn, J., K. Beattie y A. Blanchard. 2006. Seed viability and dormancy of 17 weed species after 19.7 years of burial in Alaska. Weed Science 54(3): 464-470.

13.Cousens, R. y M. Mortimer. 1995. Dynamics of weed populations. Cambridge University Press. Cambridge.

14.Delgado, M., A. Ortiz y C. Zambrano. 2006. Resistencia de Rottboellia cochinchinensis (Lour.) WD Clayton al herbicida nicosulfuron en cultivos de maíz. Agronomía Tropical 56(2): 171-182.

15.Delouche, J., N. Burgos, D. Gealy, G. Zorrilla y R. Labrada. 2007. Arroces maleza-origen, biología y control. Organización de las 
Naciones Unidas para la Agricultura y la Alimentación. Roma.

16.Dey, P., T. Pratap, S. Mishra y P. Pandit, P. 2018. Weed seed bank in soil as affected by different weed management practices in spring sweet corn. Indian Journal of Weed Science 50(3): 269-272.

17.Erasmo, E., L. Pinheiro y N. Costa. 2004. Levantamento fitosociológico das comunidades de plantas infestantes em áreas de produção de arroz irrigado cultivado sobre diferentes sistemas de manejo. Planta Daninha Viçosa-MG 22(2): 195-201.

18.Forcella, F., T. Wester y J. Cardina. 2004. Protocolos, para la determinación de bancos de semilla de malezas en los agroecosistemas. In: R. Labrada, J.C. Caseley y C. Parker. Manejo de maleza para países en desarrollo. FAO. Roma. pp. 3 -22.

19.Forte, C., L. Galon, A. Beutler, F. Basso, F. Nonemacher, F. Reichert, G. Perin y S. Tironi. 2018. Soil management systems and their effect on the weed seed bank. Pesquisa Agropecuária Brasileira 53(4): 435-442.

20.Fracchiolla, M., A. Stellacci, E. Cazzato, L. Tedone, S. Alhajj y G. De Mastro. 2018. Effects of conservative tillage and nitrogen management on weed seed bank after a sevenyear durum wheat-Faba bean rotation. Plants 7(4): 1-13.

21.Fuentes, C. 2010. Manejo de las malezas del arroz en América Latina: Problemas y soluciones. International Center for Tropical Agriculture. In: Degiovanni Beltramo, Víctor M., Martínez Racines, César P., Motta O., Francisco (eds.). Producción eco-eficiente del arroz en América Latina. CIAT, Cali. pp. 391412.

22.Gámez, A., M. Hernández, R. Díaz, R. y J. Vargas, J. 2011. Caracterización de la flora arvense asociada a un cultivo de maíz bajo riego para producción de jojotos. Agronomía Tropical 61(2): 133-140.

23.Goff, F. y G. Cottam. 1967. Gradient analysis: the use of species and synthetic indices. Ecology 48(5): 793-806.

24.Håkansson, S. 1982. Multiplication, growth, and persistence of perennial weeds. In: W. Holzner M. Numata (eds.) Biology and ecology of weeds. Springer. Dordrecht. pp. 123-135.

25.Holm, L., D. Plucknett, J. Pancho y J. Herberger. 1977. The World's Worst Weeds, distribution, and biology. The University Press of Hawaii, Honolulu.

26.Jim, C., A. Becerra, F. Aguilar, J. González, H. Solís y J. Hernández. 2020. Caracterización del banco de semillas de cuatro agroecosistemas de la Frailesca, Chiapas, México. Siembra 7(2): 93-107.

27.Keeley, P. y R. Thullen. 1971. Control of nutsedge with organic arsenical herbicides. Weed Science 19(5): 601-606.

28.Kiss, R., B. Deák, P. Török, B. Tóthmérész y O. Valkó. 2018. Grassland seed bank and community resilience in a changing climate. Restoration Ecology 26(S2): S141-S150.

29.Kissmann, K. 1997. Plantas Infestantes e Nocivas. Tomo I. Basf-Brasileira, São Bernardo do Campo.

30.Kissmann, K. y D. Groth. 1997. Plantas infestantes e nocivas. Tomo III. BasfBrasileira, São Bernardo do Campo.

31.Kissmann, K. y D. Groth. 1999. Plantas Infestantes e nocivas, Tomo II. Basf-Brasileira, São Bernardo do Campo.

32.Korres, N., J. Norsworthy, B. Young, D. Reynolds, W. Johnson, S. Conley et al. 2018. Seedbank persistence of Palmer amaranth (Amaranthus palmeri) and waterhemp (Amaranthus tuberculatus) across diverse geographical regions in the United States. Weed Science 66(4): 446-456.

33.Liebman, M, C. Mohler y C. Staver. 2001. Ecological Management of Agricultural Weeds. Cambridge University Press, New York.

34.Lorenzi, H. 2000. Manual de Identificação e de Controle de Plantas Daninhas. Plantio Direto e Convencional. Plantarum, Nova Odessa.

35.Luna, N., E. Pessoa y M. Alves. 2020. Sinopse de Marantaceae no estado de Pernambuco, Brasil. Rodriguésia 71: 1-26.

36.Mahé, I., S. Cordeau, D. Bohan, D. Derrouch, F. Dessaint, D. Millot y B. Chauvel. 2021. Soil seedbank: Old methods for new challenges in agroecology? Annals of Applied 
Biology 178(1): 23-38.

37.FAO (Organización de las Naciones Unidas para la Alimentación y la Agricultura). 2021. http://www.fao.org/faostat/ (consulta de febrero 7, 2021).

38. Ortiz, A., L. López, M. Cásares y H. Moratinos. 2009. Evaluación del banco de semilla de arroz maleza y voluntario en el suelo. Agronomía Tropical 59(4): 423-432.

39. Ortiz, A., L. Martínez, Y. Quintana, P. Pérez y A. Fischer. 2014. Resistencia de la paja Johnson [Sorghum halepense (L.) Pers.] A los herbicidas nicosulfuron $\mathrm{y}$ foramsulfuron+ iodosulfuron en Venezuela. Bioagro 26(2): 7178.

40.Partel, V., S. Kakarla e Y. Ampatzidis. 2019. Development and evaluation of a low-cost and smart technology for precision weed management utilizing artificial intelligence. Computers and Electronics in Agriculture 157: 339-350.

41.Quintero-Pertuz, I., E. Carbonó-Delahoz, E., V. Hoyos, A. Jarma-Orozco y G. Plaza. 2021. Fitosociología de malezas en plantaciones bananeras en el departamento del Magdalena, Colombia. Caldasia 43(1): 80-93.

42.Ramesh, K., A. Matloob, F. Aslam, S. Florentine y B. Chauhan. 2017. Weeds in a changing climate: vulnerabilities, consequences, and implications for future weed management. Frontiers in Plant Science 8: 95.

43. Santín-Montanyá, M., E. Zambrana-Quesada y J. Tenorio-Pasamón. 2018. Weed abundance and soil seedbank responses to tillage systems in continuous maize crops. Archives of Agronomy and Soil Science 64(12): 1705-1713.

44.Schwartz-Lazaro, L. y J. Copes. 2019. A review of the soil seedbank from a weed scientists' perspective. Agronomy 9(7): 1-1-13.

45.Sequín, L. y S. Sosa. 2019. Evolución del consumo de alimentos en Venezuela (1998-
2017). Temas de Coyuntura 78-79: 72-104.

46. Takano, H., R. Mendes, L. Scoz, R. Ovejero, J. Constantin, T. Gaines, P. Westra, F. Dayan y R. Oliveira. 2019. Proline-106 EPSPS mutation imparting glyphosate resistance in goosegrass (Eleusine indica) emerges in South America. Weed Science 67(1): 48-56.

47.Tascón, E. y A. Fischer. 1997. Malezas Específicas y Guía de Manejo. In: Pantoja, A. (ed.). MIP en Arroz: Manejo integrado de plagas: artrópodos, enfermedades y malezas. CIAT, Cali, Colombia. pp 99-116.

48.Thompson, K y J. Grime. 1979. Seasonal variation in the seed banks of herbaceous species in ten contrasting habitats. Journal of Ecology 67: 893-921.

49.Uztarroz, D. 2016. Control de Eleusine indica y Digitaria sanguinalis con herbicidas postemergentes selectivos para maíz. Instituto Nacional de Tecnología Agropecuaria (INTA). https://url2.cl/Eipvh (consulta de enero 3, 2021).

50.Vieira, S., P. Maas y F. Borchsenius. 2012. Taxonomic revision of Myrosma (Marantaceae). Blumea-Biodiversity. Evolution and Biogeography of Plants 57(2): 125-130.

51.Webster, T., T. Grey, J. Davis y A. Culpepper. 2008. Glyphosate hinders purple nutsedge (Cyperus rotundus) and yellow nutsedge (Cyperus esculentus) tuber production. Weed Science 56(5): 735-742.

52.Westwood, J., R. Charudattan, S. Duke, S. Fennimore, P. Marrone, D. Slaughter, C. Swanton y R. Zollinger. 2018. Weed management in 2050: Perspectives on the future of weed science. Weed Science 66(3): 275-285.

Zardini, E. y P. Raven. 1992. A new section of Ludwigia (Onagraceae) with a key to the sections of the genus. Systematic Botany 17(3): 481-485. 